\title{
Measuring Performance Limits of Subband Adaptive Systems
}

\author{
Alexander Stenger, Rudolf Rabenstein \\ Telecommunications Institute I \\ University of Erlangen-Nuremberg \\ Erlangen, Germany \\ \{stenger,rabe\}@nt.e-technik.uni-erlangen.de
}

\author{
Stephan Weiß, Robert W. Stewart \\ Signal Processing Division \\ Dept. of Electronic and Electrical Engineering \\ University of Starthclyde, Glasgow G1 1XW \\ $\{$ weiss,bob\}@spd.eee.strath.ac.uk
}

\begin{abstract}
We discuss a method to measure the convergence limits of general subband adaptive systems due to non-ideal filter banks. Aliasing caused in such filter banks presents a distortion to the subband adaptive system which forms a lower limit for the minimum mean squared error. The accuracy of the achievable model is given by the transfer function of the filter bank. To measure both aliasing and filter bank distortions, we employ the measurement technique by Heinle and Schiißler (1996). The presented approach is applicable to a wide range of subband adaptive filter systems. Examples for the measured limits are presented.
\end{abstract}

\section{Introduction}

Subband adaptive filtering (SAF) is widely used for problems where an adaptive system is required to identify very long impulse responses as, for example, found in acoustic echo cancellation [1, 5, 8]. The application to adaptive system identification is shown in Fig. 1, where adaptive filters operate in decimated subband signals. This has advantages over fullband realizations such as reduced computational complexity, parallelization of processing tasks, and increased convergence speed for LMS-type adaptive algorithms.

Unfortunately, SAF has several disadvantages and limitations. The effect of low energy at frequecy band edges on the convergence rate has, for example, been analysed in [6]. The achievable final minimum mean squared error (MMSE) in adaptation is limited by truncation effects and non-causality in the optimal impulse responses of the SAFs $[5,12]$. In terms of filter banks as shown in Fig. 2, the aliasing introduced in the decimation stage and the distortion of the overall filter bank system present a lower limit for both MMSE and model accuracy $[9,10]$. To design an optimally performing SAF system, an a-priori quantitative knowledge of the latter errors is therefore particularly important.

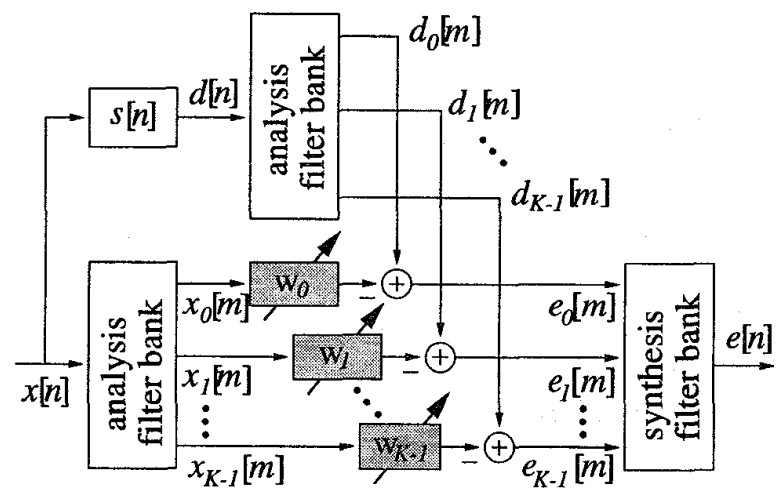

Figure 1. Subband adaptive filter

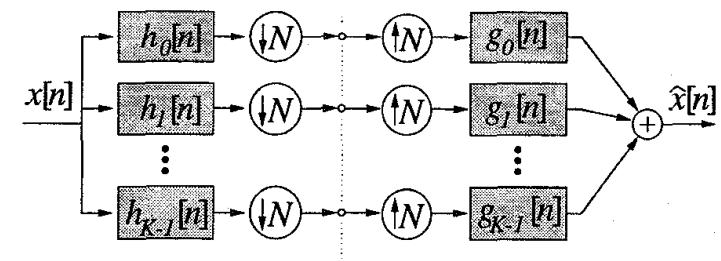

analysis filter bank synthesis filter bank

Figure 2. Analysis-synthesis system

The lower error bounds on MMSE and modelling error derived in [10] are, however, based on the use of a specific generalized DFT modulated filter bank, such that both error quantities can be expressed in terms of the coefficients of the prototype lowpass filter of the filter bank. Due to this restriction, the method can not be applied to other filter bank structures, e.g. non-uniform filter banks with different subsampling ratios [2], or to filter banks only available as "black boxes" with not explicitly known coefficients. To generalize our approach, we therefore employ the measurement method by Heinle and Schüßler [4], which measures aliasing and linear and non-linear distortions of implemented multirate systems where only the dec- 
imations/expansions rates have to be known a priori.

Based on the analytical prediction reviewed in Sec. 2, Sec. 3 outlines the principles of the proposed measurement method in. In Sec. 4, we first derive the measurement of the subband and the fullband MMSEs, and compare the results with [10]. Finally, Sec. 5 presents the measurement of the model accuracy, underlined by with practical applications.

\section{Analytical Prediction of SAF Limitations}

\subsection{MMSE Limits}

The decimation in the analysis filter bank shown in Fig. 2 can be interpreted as a linear, but time-varying operation, which can be pictured as a spectral superposition in the frequency domain. The hypothesis is, that the adaptive filter can only identify linear time-invariant (LTI) components, while alias components appear to the algorithm as noise, hence creating the MMSE under otherwise optimal adaptation conditions. Assuming the knowledge of a white noise excited source model $L_{k}\left(e^{j \Omega}\right)$ that explains all spectral shaping in the undecimated $k$ th desired subband $d_{k}[m]$ by including a noise shaping filter for $x[n]$, the unknown system $S\left(e^{j \Omega}\right)$, and the transfer function of the $k$ th analysis filter $H_{k}\left(e^{j \Omega}\right)$, the alias spectrum in $d_{k}[m]$ can be isolated

$$
L_{k}^{\text {alias }}\left(e^{j \Omega}\right)=\sum_{\nu=0}^{N-1} L_{k}\left(e^{j\left(\frac{\Omega}{N}+\frac{2 \pi}{N} \nu\right)}\right) \cdot W_{k}\left(e^{j\left(\frac{\Omega}{N}+\frac{2 \pi}{N} \nu\right)}\right)
$$

where $W_{k}\left(e^{j \Omega}\right)$ is a rectangular window that cancels the passband and transition bands of the $k$-th analysis filter. The window is symmetrical to the filter's center frequency $\Omega_{c, k}$ and $\frac{2 \pi}{N}$ wide:

$W_{k}\left(e^{j \Omega}\right)= \begin{cases}0 & \Omega_{c, k}-\frac{\pi}{N}<\Omega+2 \pi i<\Omega_{c, k}+\frac{\pi}{N}, i \in \mathbb{N} \\ 1 & \text { elsewhere }\end{cases}$

From the alias spectrum in (1) we can calculate the aliascomponent PSD, which forms a lower bound for the the subband error signal PSD:

$$
S_{e_{k} e_{k}}^{\text {alias }}\left(e^{j \Omega}\right)=\left|L_{k}^{\text {alias }}\left(e^{j \Omega}\right)\right|^{2} .
$$

Finally, the fullband error PSD is obtained by applying the subband error signals to the synthesis filter bank:

$$
S_{e e}^{\text {alias }}\left(e^{j \Omega}\right)=\left|\sum_{k=0}^{K-1} L_{k}^{\text {alias }}\left(e^{j N \Omega}\right) \cdot G_{k}\left(e^{j \Omega}\right)\right|^{2}
$$

where $G_{k}\left(e^{j \Omega}\right)$ is the transfer function of the $k$-th synthesis filter as shown in Fig. 2. Finally, the minimum variance of the fullband error signal is calculated from (4) using the Wiener-Khintchine transform,

$$
\operatorname{MMSE}=\frac{1}{2 \pi} \int_{-\pi}^{\pi} S_{e e}^{\text {alias }}\left(e^{j \Omega}\right) d \Omega .
$$

If no information on the spectral characteristics of the signal $x[n]$ and the unknown system $s[n]$ is available, alternatively a source model $L_{k}\left(e^{j \Omega}\right)$ only consisting of the analysis filters may be used. The error introduced by this approximation is usually tolerable, as experiments in [10] demonstrate.

\subsection{Model Accuracy}

The equivalent fullband model of the SAF system shown in Fig. 1 is given by the overall impulse response of the transfer path between $x[n]$ and $e[n]$ with adapted subband filters. If aliasing is sufficiently suppressed in the subbands (as mandatory for low MMSE), the accuracy of the equivalent fullband model is given by deviation of the filter bank distortion function

$$
T\left(e^{j \Omega}\right)=\sum_{k=0}^{K-1} H_{k}\left(e^{j \Omega}\right) G_{k}\left(e^{j \Omega}\right)
$$

from unity [10].

\section{Measurement Method of Heinle/Schüßler}

The measurement method by Heinle and Schüßler [4] allows to isolate a number of different error sources in multirate systems. It is based on an implicit polyphase representation of the system to be measured, whereby the multirate system is split into a linear periodically time-varying multiplexer part, and a multiple input-output system consisting of LTI transfer functions. These LTI transfer functions can be determined, and can be separated from any potential nonlinearities (e.g. due to quantization) or other noise sources within the system.

Fig. 3 shows the application to one branch of an analysis filter bank, $L_{k}(z)$. The test signal $u[n]$ is a random phase signal which avoids zeroes in the magnitude spectrum. In our experiments we use uniformly distributed white noise generated with MATLAB. The output signal $d_{k}[m]$ has a lower sampling rate than the input signal $u[n]$. The measurement program uses both the input and output signal of the implemented system to calculate $N$ separate LTI transfer functions $L_{k, \nu}\left(e^{j \Omega}\right), \nu \in[0 . . N-1]$ at $M$ frequency points $\Omega_{\mu}=\mu \cdot \frac{2 \pi}{M}$. A standard measurement method for LTI-Systems is used that yields unbiased and consistent spectral estimates and isolates other distortions [3]. For convenience we will denote the measured transfer functions with $L_{k, \nu}(\mu)$. Each transfer function $L_{k, \nu}(\mu)$ characterizes the corresponding alias component in the $k$-th subband. 


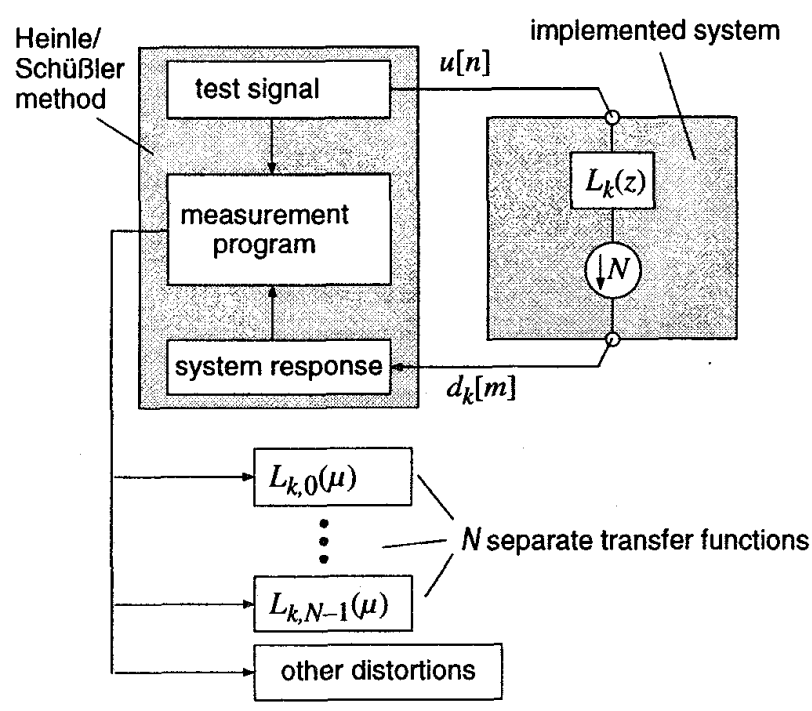

Figure 3. Measurement example with the Heinle/Schüßler method

We can reconstruct the frequency response of the bandpass $L_{k}(\mu)=L_{k}\left(e^{j \frac{2 \pi}{N M} \mu}\right)$ before downsampling by simply concatenating the measured components

$$
L_{k}(\mu)=\sum_{\nu=0}^{N-1} \tilde{L}_{k, \nu}(\mu-\nu M), \mu \in[0 . . N M-1]
$$

where

$$
\tilde{L}_{k, \nu}(\mu)=\left\{\begin{array}{cc}
L_{k, \nu}(\mu) & \mu \in[0 . . M-1] \\
0 & \mu \notin[0 . . M-1]
\end{array}\right.
$$

Note that the bandpass transfer function $L_{k}(\mu)$ is represented by $N \cdot M$ frequency points, while the $N$ times downsampled components $L_{k, \nu}(\mu)$ have only $M$ frequency points. Fig. 4 illustrates this operation, which can be interpreted as a reverse downsampling procedure.

This demonstrates the advantage of the measurement method: even without any explicit information about the filter bank other than the decimation rate, we can obtain a frequency-domain description of each bandpass. Note that this is possible even although only a downsampled output signal is available.

\section{Measuring the PSD of Alias Components in the Error Signals}

This section describes how we can determine a lower limit for error signals from the aliasing introduced by a "black box" analysis filter bank by applying the method outlined in Sec. 3. To perform this calculation, the center frequency $\Omega_{c}$ and the downsampling factor $N$ in each subband

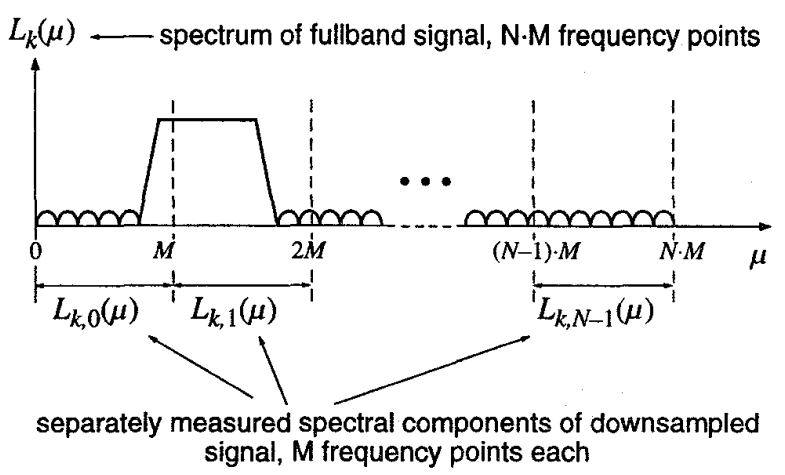

\section{Figure 4. Concatenating measured spectra to fullband spectrum}

must be known. In section 4.1 limits for the subband error signals are derived, and in section 4.2 we calculate the PSD and the variance of the fullband MMSE signal, where the synthesis filter bank is assumed to be another implemented "black box".

\subsection{Subband Error Signals}

Fig. 5 shows the measurement setup. The analysis filter bank must be an implemented system, either in software or hardware (e.g. a DSP realization). Ideally, the spectral characteristics of of both the input signal, $x[n]$ and the unknown system $s[n]$ should be known. They can be exploited to realize a source model, which is used to preprocess the input $d[n]$ of the filter bank.

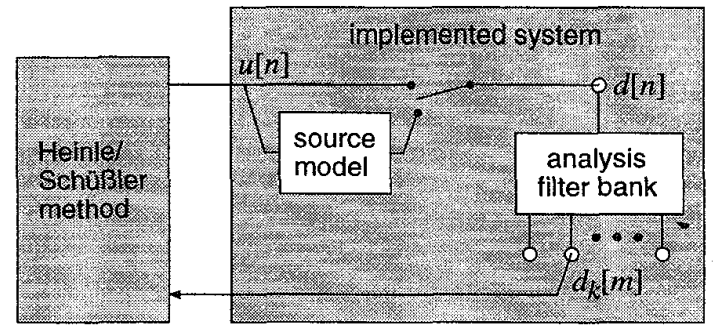

\section{Figure 5. Measurement of subband error sig- nal spectrum}

The measurement program applies a random test signal $u[n]$ to the device under test and records the decimated system response $d_{k}[m]$. Based on this, the Heinle/Schüßler method determines $N$ transfer functions $L_{k, \nu}(\mu)$ as illustrated in Fig. 3. If the $L_{k, \nu}(\mu)$ are assembled according to (7) and (8), we obtain an $M \cdot N$ point frequency spectrum 
$L_{k}(\mu)$. At this point we proceed as in section 2, using sampled versions of (1)-(3). Note that spectra of subsampled signals have $M$ frequency points, while fullband signals are represented by $N \cdot M$ point spectra.

The PSD of the $k$-th subband MMSE signal is given by

$$
\begin{array}{r}
S_{c_{k} e_{k}}^{\text {alias }}(\mu)=\left|\sum_{n=0}^{N-1} L_{k}(\mu+n M) W_{k}(\mu+n M)\right|^{2}, \\
\mu \in[0 . . M-1],
\end{array}
$$

where $W_{k}(\mu)$ equals (2), sampled at $N \cdot M$ frequency points $\Omega=\frac{2 \pi}{N M} \mu$. From (9) the minimum subband error variance can be obtained by an inverse Wiener-Kintchine transform,

$$
\operatorname{MMSE}_{\mathrm{k}}=\frac{1}{M} \sum_{\mu=0}^{M-1} S_{e_{k} e_{k}}^{\text {alias }}(\mu)
$$

\subsection{Fullband Error Signal}

Using the Heinle/Schüßler method, an implemented synthesis filter bank can be measured similarly to the analysis filter bank as described in Sec. 3. Thereby, $k$ th bandpass $G_{k}(\mu)$ of the synthesis bank can be assembled according to Fig. 4 from $N$ separately measured transfer functions, where $N$ is te upsampling factor.

Once the alias spectra $L_{k}^{\text {alias }}(\mu)$ and the transfer functions of the synthesis filters, $G_{k}(\mu)$ are known, it is straightforward to calculate the minimum error PSD $S_{e e}^{\text {alias }}(\mu)$ of the output signal $e[n]$ by applying (4), evaluated at $N \cdot M$ frequency points, i.e. let $\Omega=\frac{2 \pi}{N M} \mu$ in (4). Finally as in (10), the fullband MMSE is obtained by an inverse WienerKintchine transform,

$$
\operatorname{MMSE}=\frac{1}{M} \sum_{\mu=0}^{M-1} S_{e e}^{\text {alias }}(\mu)
$$

\subsection{Practical Examples}

To demonstrate the prediction of error limits, in a first example we compare the measured fullband error PSD against simulated results of a system identification with a $K=8$ channel GDFT filter band decimated by $N=7$. The simulation was performed using a complex valued NLMS algorithm with a sufficient number of adaptive filter coefficients, a small stepsize (0.05), and white noise excitation. For illustration purposes, a system to be identified contained a very dominant pole-pair at $\Omega=\frac{\pi}{12}$. The grey curve in Fig. 6 shows the PSD of the residual fullband error signal after $2 \cdot 10^{5}$ iterations. A prediction of the minimum error PSD was performed according to Secs. 4.1 and 4.2 on the same system, and is shown in Fig. 6 as a black curve. Apparently, it forms a tight lower bound for the residual

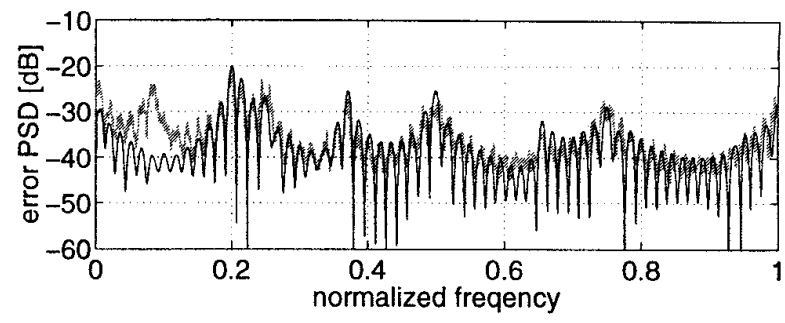

Figure 6. simulated and measured fullband error PSD

error PSD obtained by simulation. Differences between predicted and simulated curve (particularly in the lowpass band, $\Omega=0 \ldots \frac{\pi}{4}$ ) are due to insufficient adaptation around the original signal's peak and at band egdes, where large spectral dynamics cause the NLMS algorithm to converge only slowly $[11,6]$.

The second example will illustrate the MMSE prediction using an acoustic echo cancelling application. The unknown system is a room impulse reponse with a length of 2000 coefficients, which has to be identified by an SAF system using a $K=32$ band GDFT filter bank. For a number of different decimation ratios $N$, the filter banks were optimized for acoustic echo cancellers [7]. Tab. 1 compares the analytically calculated fullband MMSE (Sec. 2) with the measured values (Sec. 4). For the upper two rows in Tab. 1, MMSE predictions based on both analytic and measured methods are obtained with a white source model (s. m.), while for the next two a spectral model of the room response was employed as source model. The last row shows simulation results with the NLMS algorithm using a large step size $(0.3)$ and a sufficient number of adaptive coefficients. The MSE was evaluated after the adaptive fiters had converged.

Table 1. fullband error variance (in $\mathrm{dB}$ ) of an SAF with GDFT filter bank

\begin{tabular}{|l|c|c|c|c|c|c|}
\hline$N$ & 30 & 29 & 28 & 27 & 26 & s. $\mathrm{m}$. \\
\hline \hline calc. & 31.04 & 39.57 & 36.21 & 47.10 & 57.00 & no \\
\hline meas. & 31.08 & 39.46 & 36.23 & 47.13 & 57.00 & no \\
\hline \hline calc. & 33.75 & 38.58 & 38.22 & 51.13 & 49.45 & yes \\
\hline meas. & 33.78 & 38.53 & 38.19 & 51.15 & 49.44 & yes \\
\hline \hline sim. & 29.5 & 34.2 & 36.0 & 36.1 & 37.0 & \\
\hline
\end{tabular}

The measured results favourably compare to the analytically predicted ones, and form are a lower bound for the simulation results. For $N \geq 28$ the difference between simulation and predictions is mainly due to the large step size which leads to an excess error [11]. For $N \leq 27$ other effects than aliasing limit the final error. In a practical system design, this result suggests not to choose the filter banks 
with $N \leq 27$, as a further reduction of aliasing does not improve the overall SAF performance significantly.

\section{Measuring Model Accuracy}

In Sec. 2.2 we have discussed how the distortion of the filter banks limits the model accuracy of the adapted SAF system. In the following, we introduce a method to perform this analysis by measuring an already implemented system.

If an implemented analysis-synthesis filter bank system as given in Fig. 2 exhibits a sufficiently low aliasing level in the subbands (which may be checked using the measurement procedures introduces in Sec. 4), the overall filter bank appears as an LTI system and can be characterized by a single transfer function, $T\left(e^{j \Omega}\right)$. This transfer function can again be assembled from measured components using the Heinle/Schüßler method, following the approach outlined in Fig. 4. Its discretely sampled version, $T(\mu)$, can now be used to calculate the error in power complementarity,

$$
\mathrm{RE}=\frac{1}{M} \sum_{\mu=0}^{M-1}(1-|T(\mu)|)^{2} .
$$

which gives the lower limit in accuracy for the modelling capability of the SAF system in form of a quadratic error term at $M$ frequency points $\Omega_{\mu}=\frac{2 \pi}{M} \mu$. It should be noted that (12) mainly considers amplitude distortion which is sufficient for linear phase filter bank systems. Otherwise, (12) also has to accommodate for phase distortion.

To verify the measured error limit in modelling accuracy, we compare it with both simulations and the analytical prediction method in [10] for a number of different filter banks in Tab. 2. For the simulation part, equivalent fullband models have been reconstructed from adapted filters in an RLS system identification set-up. The reconstruction error is given by the reconstructed model and the "unknown" system $s[n]$. Obviously, analytical and measured limits are

Table 2. Model reconstruction error (in [dB]) of an SAF with GDFT filter bank

\begin{tabular}{|l|c|c|c|c|c|}
\hline$N$ & 30 & 29 & 28 & 27 & 26 \\
\hline \hline calc. & -24.54 & -44.26 & -29.81 & -63.94 & -41.43 \\
\hline meas. & -24.55 & -44.26 & -29.81 & -63.94 & -41.43 \\
\hline sim. & -23.28 & -43.14 & -28.80 & -63.65 & -41.04 \\
\hline
\end{tabular}

virtually identical and form a tight lower bound for the modelling error attained in the simulations.

\section{Conclusions}

We have presented an accurate prediction of the lower limit for adaptation of subband adaptive filter systems based on aliasing measurements of the employed filter bank. Our measurement procedure does not require an explicit knowledge of the filter banks and can even be applied to implemented systems. The appeal is that for SAF applications such as acoustic echo cancellation, the performance limits imposed by given filter banks can be easily verified without time-consuming simulations.

\section{References}

[1] A. Gilloire and M. Vetterli. "Adaptive Filtering in Subbands with Critical Sampling: Analysis, Experiments and Applications to Acoustic Echo Cancelation". IEEE Transactions on Signal Processing, Vol.SP-40(No.8):pp.1862-1875, Aug. 1992.

[2] M. Harteneck, J. M. Páez-Borrallo, and R. W. Stewart. "An Oversampled Subband Adaptive Filter Without Cross Adaptive Filters". Signal Processing, Vol.64(No.1):pp.93-101, January 1998.

[3] F. Heinle, R. Rabenstein, and A. Stenger. "A Measurement Method for the Linear and Nonlinear Properties of Electro-Acoustic Transmission Systems”. Signal Processing, Vol.64(No.1):pp.49-60, January 1998.

[4] F. A. Heinle and H. W. Schüßler. "Measuring the Performance of Implemented Multirate Systems". In Proc. IEEE International Conference on Acoustics, Speech, and Signal Processing, pages 2754-2757, Atlanta, GA, May 1996.

[5] W. Kellermann. "Analysis and Design of Multirate Systems for Cancellation of Acoustical Echoes". In Proc. IEEE International Conference on Acoustics, Speech, and Signal Processing, volume 5, pages 2570-2573, New York, 1988.

[6] D. R. Morgan. "Slow Asymptotic Convergence of LMS Acoustic Echo Cancellers". IEEE Transactions on Speech and Audio Processing, Vol.2(No.3):pp.126-136, March 1995.

[7] S. Weiß, M. Harteneck, and R. W. Stewart. "On Implementation and Design of Filter Banks for Subband Adaptive Systems". In IEEE Workshop on Signal Processing Systems (SiPS'98), Cambridge, MA, October 1998.

[8] S. Weiß, U. Sörgel, and R. W. Stewart. "Computationally Efficient Adaptive System Identification in Subbands with Intersubband Tap Assignment for Undermodelled Problems". In Asilomar Conference on Signals, Systems, and Computers, pages 818-822, Monterey,CA, November 1996.

[9] S. WeiB, A. Stenger, R. Rabenstein, and R. W. Stewart. "A Lower Error Bound for Oversampled Subband Adaptive Systems". IEE Electronics Letters, Vol.34(No.16):pp.15551557, August 1998.

[10] S. Weiß, R. W. Stewart, A. Stenger, and R. Rabenstein. "Performance Limitations of Subband Adaptive Filters". In European Signal Processing Conference, volume III, pages 1245-1248, Rodos, Greece, September 1998.

[11] B. Widrow and S. Stearns. Adaptive Signal Processing. Prentice Hall, Englewood Cliffs, New York, 1985.

[12] R. J. Wilson, P. A. Naylor, and D. Brookes. "Performance Limitations of Subband Acoustic Echo Controllers". In Proc. International Workshop on Acoustic Echo and Noise Control, pages 176-179, Imperial College, London, UK, September 1997. 